\title{
Editorial
}

\section{Plastic surgery: What is new and what is still under wraps?}

T: he answer for the question "what is a recent advance" really depends on what subject one is referring to. Whereas, in Anthropology anything that happened in the last few centuries is recent, in the fashion world what was on the ramp last evening is obsolete today. Plastic surgery thankfully is somewhere in between, and though it is a fast growing superspeciality, what boggles the mind is the numerous avenues in which it is showing progress. I put this forward march of plastic surgery under "4 Rs" - Repair, Reanimate, Rejuvenate and Regenerate and and in each sub-speciality of Plastic Surgery we come across path breaking recent developments.

\section{CONGENITAL ANOMALIES}

In the area of congenital anomalies, the thrust is in the understanding of the occurrence of these anomalies, research on long-term outcomes, genes and their interaction with other genes and with the environment, and are all aimed at identifying their cause of occurrence and thereby preventing them in the future. Epidemiological studies are disturbingly suggesting that individuals born with clefts have a shorter life span and are more prone to develop cancers and psychological disorders..$^{[1,2]}$ Is this then going to be the new frontier of research in clefts?

Advancements in the various multidisciplinary fields involved in cleft management have substantially improved functional and aesthetic outcomes. The legitimacy of such controversial topics such as gingivoperiosteoplasty, primary rhinoplasty, and presurgical nasoalveolar molding is heavily contested. Bone morphogenetic protein and other recombinant growth factors may play important

\begin{tabular}{|l|l|}
\hline \multicolumn{3}{|c|}{ Access this article online } \\
\hline Quick Response Code: & Website: \\
\hline & www.ijps.org \\
\cline { 2 - 2 } & Dol: \\
\hline
\end{tabular}

roles in future cleft care. Translational research from fields such as fetal wound healing, tissue engineering, and gene therapy may have clinical applications as cleft care continues to evolve.

\section{CRANIO-MAXILLOFACIAL SURGERY}

Technological advances in materials and biological sciences and improved surgical techniques have remarkably improved clinical outcomes in Craniomaxillo-facial surgery. The quest to raise the bar for patient care continues to inspire advances for predictable biological regeneration of soft and hard tissues. But then, is the use of recombinant DNA engineered biologicals daring, or for that matter sensible? Is it logical, timely and safe? It is crucial for the practicing craniofacial surgeon to take a step back periodically and carefully review the biological factors that have the potential for dramatically altering the discipline of craniofacial surgery. The growth factor technology underscoring bone tissue regeneration is an interesting frontier and as the $21^{\text {st }}$-century matures, recombinant human biologicals will have an overwhelming impact on the practice of craniofacial surgery.

Distraction osteogenesis (DO) is a relatively new method of treatment for selected deformities and defects of the oral and facial skeleton. Although first used in 1903 and popularised in the 1950s by the Russian orthopaedic surgeon, Dr. Gabriel Ilizarov for correcting bony deformities and repair defects of the arms and legs, we, plastic surgeons, used the concept in the facial skeleton only recently to treat TMJ ankylosis, hemifacial microsomia and as a substitute of alveolar bone grafting in clefts. During the first year of life, periosteal bone regeneration may allow lengthening without corticotomy. The main limitations of DO of the craniofacial skeleton have been technical, with bulky and cumbersome external appliances, but newer designs of multi-planer miniature distraction devices have been successfully applied to achieve mandibular, maxillary, and orbital expansion.

Indian Journal of Plastic Surgery January-April 2012 Vol 45 Issue 1 


\section{MICROSURGERY AND RECONSTRUCTIVE SURGERY}

Microsurgery has entered a bold new frontier of 'super micro-surgery'. The technique to anastomose super-micro vessels has been established through the development of high-resolution operating microscopes and supermicro instruments and sutures based on the advanced industrial technology of Japan. This has driven forward vascular surgery to treat paediatric finger tip replants and perform microlymphatic surgery for lymphoedema in carcinoma breast by autologous lymph node transplant from the femoral region to the axilla.

Perforator flaps represent the most recent advance in the development of free flap surgery. These flaps are based on perforating vessels and can be harvested without significant damage to associated muscles, thereby reducing the postoperative morbidity associated with muscle-based flaps. Elevation of perforator flaps requires a meticulous technique and can be more challenging than raising muscle-based flaps. Use of a Doppler device enables reliable identification of the perforating vessels and aids in the design of free-style free flaps, where the flaps are designed purely according to the perforator located. The major advantage of free-style free flaps is that an unlimited number of flaps can potentially be designed on much shorter pedicles. The anterolateral thigh flap is the most commonly used perforator flap in head and neck reconstruction and has virtually replaced the radial forearm flap and thus the latter's donor site morbidity.

Functional muscle transfer for both facial palsy and brachial plexus injury are being refined constantly. Results in both obstetric brachial plexus injury and traumatic ones are improving every day and regenerative medicine may make things even better in the future.

Composite tissue allotransplantation (CTA) — face transplant or hand transplant adheres to the tried and tested trends of organ transplantation: harvesting from the donor in the dead cerebral status, immunological incompatibility between donor and recipient, and life-long immunosuppression of the recipient. CTA is a tremendous hope for reconstructive surgery but is still so rarely performed that it invariably becomes 'Breaking News' even today! Although the results of the first clinical cases are reported to be encouraging to very exciting in some cases, continued success in clinical CTA over the next few years could convince the transplant community to shed its skepticism that all attempts at CTA are ambitious and misguided. Tolerance induction, allowing minimizing the immunosuppression, seems a realistic milestone to reach in the coming years, enlarging the indications of allotransplantation to mutilations less severe than bilateral hand amputation and chemical burns or gun-shot injuries of face. Meanwhile, restricted to major handicap conditions and performed under the fundamental guidelines of medical ethics-professional competency, therapeutic objective, and information of the patient(CTA) is a rightful expression of reconstructive surgery.

\section{HAND}

The spiral CT-scan technology has revolutionised our diagnostic techniques, allowing 3D visualisation of complex fractures and dislocations, and demonstrating bone healing after osteosynthesis of complex hand fractures and nonunions. Three-dimensional imaging will be probably shortly coupled to kinematic analysis, to better understand for example the normal and abnormal motion of the shoulder joint or carpal bones. New MRI sequences allow better definition of hyaline cartilage. Diffusion tensor tractography will permit very soon functional imaging of peripheral nerves. Biological enhancement of bone and tendon healing are currently under evaluation. Computer-assisted surgery could be quite useful in selective indications of arthroplasty or bone fixation; and a robot could be our future assistant in the operative room.

In the field of peripheral nerve surgery, the techniques of nerve repair have progressed and some immunosuppressive agents could as well be beneficial to enhance axonal regeneration. Peripheral neurotisations provide early motor or sensitive recovery after brachial plexus injuries. Neurotisation of proximal muscles is also now used in amputees, for better motor control of the prosthesis improve dexterity and even to provide some sensibility. Indeed, there are currently many advances in upper extremity prosthetics. The techniques of physiotherapy (based for example on cognitive neurosciences) and of objective functional evaluation have as well progressed in recent years.

\section{BURNS}

A burn injury today is recognised as a dysfunction of almost every cell in the body and various organs leading 
to alterations, not only in cellular functions but also in cellular signaling pathways. The current thought that alterations and new treatments of these pathways may restore cellular function and productivity, which in turn may lead to an improved energy metabolism, increase cell survival, with eventual lowering of morbidity, and mortality, is perhaps the most cutting-edge research in burn care. Gene therapy is another emerging technique to alter dermal and epidermal regeneration and to improve wound healing. Non-viral constructs have been shown to effectively change the cells into a productive and active cell that releases growth factors and other signaling factors to improve dermal and epidermal regeneration. Liposomal gene constructs have been shown to transfect dermal stem cells increasing the therapeutic possibilities of this futuristic technology!

Cultured skin substitute (CSS), which is the patient's own epithelium in combination with a dermal substitute (Integra) represents a full-skin substitute including both dermis as well as epidermis. The use of bilayer artificial skin has improved the survival and cosmetic results of early eschar excision in patients with massive full-thickness burns. Recent advances in burn care has seen improvement in acute care, such as resuscitation and management of inhalation injuries, early excision and grafting, the control of infections and improving the immune system, improvements in provision of metabolic and nutritional requirements, evolution of effective skin banks, infection control, and alternative wound-closure materials and strategies. The use of tissue expanders to assist our reconstructive efforts is also a boon to burn scar management.

\section{MINIMAL ACCESS PLASTIC SURGERY}

Plastic surgeons have not been left behind in this race of smaller scars and lesser morbidity and improvements in technology have enabled us to use endoscopy for many cosmetic procedures, including facelifts, abdominoplasty, and breastaugmentation. Endoscopicfacial surgeryincluding Endo-Forehead/Brow Lift, Endo-Blepharoplasty, EndoCantopexy, Endo-Peri-Orbital rejuvenation, Endo-Mid-Face lift and Endoscopic Malar Augmentation (transtemporal) are being practiced in many units. Endoscopic management of facial fractures which are difficult to access by conventional routes also represent a quantum leap in use of this concept. Two examples of this are the subcondylar fracture management, and orbital wall access.
Endoscopy can also be used in other reconstructive procedures such as harvesting free flaps, harvesting tissues like the omentum, jejunum, temporoparietal fascia, tensor fascia lata, sural nerve, intercostals nerve, rib cartilage, vein grafts and also to place tissue expanders and releasing carpel tunnels. Other conditions which are treated by minimal access include gynaecomastia, lipoma excision, torticollis, putting static slings in facial palsy, correction of masseteric hypertrophy by the intraoral approach, decompression fasciotomy, perforator vein surgery for varicosity, retrieval of severed tendon, and pectus excavatum.

\section{AESTHETIC SURGERY}

Advances in both technology and knowledge today have taken aesthetic surgery to a whole new horizon. Improvements in computerised delivery systems have led to a wider field of application of lasers in surgery and more predictable results. Thus selective photothermolysis with lasers allows removal of certain pigmented lesions and capillary vascular malformations with minimal nonspecific skin damage, and the pulsed carbon dioxide laser allows precise dermabrasion for facial rejuvenation. The wavelength of $10,600 \mathrm{~nm}$, which is absorbed by the water in cell cytoplasm, combined with a pulse of less than a millisecond, causes little non-specific injury.

Nutrition is an ever-changing dynamic field filled with jargon, scientific facts and plenty of myths from detox to fat-burners to food combining. Some science needs to percolate through this look-young industry, lest it gets clubbed with quackery. A bunch of 'anti aging medicines' are also desperately trying to acquire scientific acceptance and we can use them only after they have undergone proper trials.

In breast surgery the spectra adjustable gel implant is a new dual-lumen gel implant, developed by Becker in 2006, has a small inner chamber that can be filled with saline while the outside chamber is a gel implant. If, postsurgically, further volume is needed, the inner lumen can be filled to the desired volume before the fill tube is removed. If increased projection is desired, the inner lumen can be overfilled. Projection can thus be increased without decreasing the base diameter.

\section{REGENERATIVE MEDICINE}

Regenerative medicine is a whole new world, which Indian Journal of Plastic Surgery January-April 2012 Vol 45 Issue 1 
still needs to be explored and understood. It is a grand combination of biomaterials, growth factors and stem cells, to repair failing and injured organs. Material scientists can now fabricate biocompatible scaffolds with a wide range of physical parameters, combining mechanical integrity with high porosity to promote cell infiltration and angiogenesis. Likewise, biochemists can produce highly purified, bioactive cytokines in large quantity, suitable for cell culture and in vivo applications. Adipocyte-derived stem cells are the current toast of scientists as there is a much larger concentration of stem cells and precursor cells in fat when compared to bone marrow, which is the other well-researched source. Adipose tissue represents an abundant and accessible source of adult stem cells with the ability to differentiate along multiple lineage pathways. Stem cell therapy is in clinical and therapeutic trial stages in radiation necrosis, breast reconstruction, breast augmentation, facial aesthetic surgery, tracheal repair, calvarial repair, tendon and muscle repair.

Better understanding of the cellular biology of these tissues has allowed regeneration to produce bone, cartilage, and epidermis. Thus instead of carving out the cartilaginous framework of external ear from cosal cartilage confluence, we now can seed human chondrocytes on a vicryl scaffold to generate the neocartilage with matrix. Performed in the nude mouse, which is not immuno-competent, clinical studies applying this technology are awaited!

Human keratinocyte culture can vastly expand skin autografts and are the obvious answer to extensive burns. This has only partly solved the clinical problem as it takes three weeks to produce a large confluent sheet of cultured keratinocytes, and without dermal support the keratinocyte graft is relatively unstable. Allogenic dermis, from which the highly antigenic epidermis has been dermabraded, has been used on the wound bed initially and subsequently after $2-3$ weeks the cultured autologous keratinocytes can be used to cover it. A cultured skin substitute includes the use of collagen matrices seeded with cultured fibroblasts and covered with autologous keratinocytes. Thus we are actually taking steps towards "off the shelf skin replacement" in the future.

\section{WOUND HEALING}

Plastic surgeons are in the forefront of 'Scarwars' and are fighting scars to prevent disfigurements and deformities. A sequence of growth factors and cytokines released after injury controls wound healing. In foetuses, wounds heal with a reduced inflammatory and angiogenic response and without scarring. By manipulating the concentrations of transforming growth factor $\beta$ (TGF $\beta$ ) in wounds in adult human skin, scar formation can be reduced. Applying this in clinical practice will surely be yet another research frontier.

'Regulated negative pressure therapy' and more recently 'regulated oxygen + negative pressure therapy' have converted open wounds into closed wounds and assisted in wound healing by assisting tissue granulation in wound, promoting tissue perfusion, maintaining a moist atmosphere, removing infectious material, helping healing of wounds with tunneling and undermining and applying controlled, localised negative pressure to help draw wound edges together. The negative pressure also reverses the lymphatic flow thus localizing the infection and also assists in graft take when applied over the graft. The oxygen prevents anaerobic infection by raising the $\mathrm{PO} 2$ of the wound.

I am quite sure there are many other frontiers in Plastic Surgery, which have not crossed my mind, and there are many more I am not even aware of, but with these few that I can remember, it surely has been an exiting recent past and we are undoubtedly moving towards an even more exhilarating future.

\section{Surajit Bhattacharya \\ Editor, IJPS \\ E-mail: surajitbh@yahoo.co.in}

\section{REFERENCES}

1. Christensen K, Juel K, Herskind AM, Murray JC. Long term follow up study of survival associated with cleft lip and palate at birth. BMJ 2004;328:1405.

2. Zhu JL, Basso O, Hasle H, Winther JF, Olsen JH, Olsen J. Do parents of children with congenital malformations have a higher cancer risk? A nationwide study in Denmark. Br J Cancer 2002;87: 524-8.

How to cite this article: Bhattacharya S. Plastic surgery: What is new and what is still under wraps?. Indian J Plast Surg 2012; 45:1-4. 